\title{
Hospital cost and clinical effectiveness of robotic-assisted versus video-assisted thoracoscopic and open lobectomy: $A$ propensity score-weighted comparison
}

\author{
Peter J. Kneuertz, MD, ${ }^{a}$ Emily Singer, MD, ${ }^{\text {a }}$ Desmond M. D'Souza, MD, ${ }^{\text {a }}$ \\ Mahmoud Abdel-Rasoul, MS, MPH, ${ }^{\text {b }}$ Susan D. Moffatt-Bruce, MD, PhD, MBA, and \\ Robert E. Merritt, MD
}

\section{ABSTRACT}

Objective: To compare cost and perioperative outcomes of robotic, videoassisted thoracoscopic surgery (VATS), and open surgical approaches to pulmonary lobectomy.

Methods: Patients who underwent pulmonary lobectomy between 2012 and 2017 at a single tertiary referral center were reviewed. Propensity score adjustment by inverse probability of treatment weighting (IPTW) was used to balance baseline patient characteristics. The primary outcomes of the study were direct hospital cost and perioperative outcomes, including operative time, complications rates, and length of stay. Indirect cost and charges were secondary financial outcomes.

Results: A total of 697 patients underwent pulmonary lobectomy by robotic $(\mathrm{n}=296)$, VATS $(\mathrm{n}=161)$, and open thoracotomy $(\mathrm{n}=240)$. In the IPTWadjusted analysis, open thoracotomy had the shortest mean operating room time (robotic 278 minutes vs VATS 298 minutes vs open 265 minutes, $P=.05$ ), and lowest operating room costs (robotic $\$ 9,912$ vs VATS $\$ 9491$ vs open $\$ 8698$, $P=.001)$. Length of stay was significantly shorter after robotic and VATS lobectomy (robotic 3.8 days vs VATS 3.8 days vs open 5.4 days, $P<.001$ ), with significantly fewer events of atelectasis and pneumonia as compared with the open group. In sum, no significant differences were seen in IPTW-adjusted direct cost (robotic $\$ 17,223$ vs VATS $\$ 17,260$ vs open $\$ 18,075, P=.48$ ), indirect cost, or charges for the total hospital stay.

Conclusions: Robotic and VATS lobectomy were associated with similar cost and improved clinical effectiveness as compared with the open thoracotomy approach. Increased procedural cost of minimally invasive lobectomy can be recovered by postoperative costs reductions, associated with improved postoperative outcomes and shorter hospital stay. (J Thorac Cardiovasc Surg

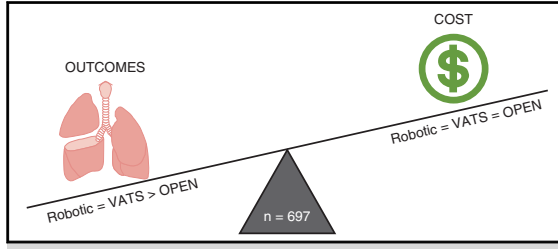

Value consideration of robotic, VATS, and open pulmonary lobectomy.

\section{Central Message}

Minimally invasive lobectomy is associated with superior clinical outcomes and similar hospital cost as compared with open lobectomy, with no significant difference between the robotic and VATS approach.

\section{Perspective}

In a propensity score-weighted analysis, we have found no significant difference in total cost to the hospital between robotic, VATS, and open lobectomy. Greater procedural cost of minimally invasive lobectomy was recovered in the postoperative period. The improved clinical outcomes of both robotic and VATS compared with open lobectomy are important when considering the overall value of minimally invasive procedures.

See Commentary on page 2027. 2019;157:2018-26)

Pulmonary lobectomy remains the most commonly performed major operation in general thoracic surgery practice. Over the last 2 decades, there has been a substantial

From the ${ }^{\mathrm{a}}$ Thoracic Surgery Division, Department of Surgery, The Ohio State University Wexner Medical Center, Columbus, Ohio; and ${ }^{\mathrm{b}}$ Center for Biostatistics, The Ohio State University, Columbus, Ohio.

Received for publication Aug 9, 2018; revisions received Dec 6, 2018; accepted for publication Dec 27, 2018; available ahead of print Feb 26, 2019.

Address for reprints: Peter J. Kneuertz, MD, Thoracic Surgery Division, Department of Surgery, The Ohio State University Wexner Medical Center, Doan Hall N846, 410 West 10th Ave, Columbus, OH 43210 (E-mail: Peter.Kneuertz@osumc.edu). $0022-5223 / \$ 36.00$

Copyright (c) 2019 by The American Association for Thoracic Surgery

https://doi.org/10.1016/j.jtcvs.2018.12.101 evolution in surgical techniques. Currently, there are multiple established approaches that are all frequently performed. In the most recent nationwide report of lobectomy for lung cancer from the National Cancer Database, the majority of lobectomies, up to year 2012, were performed via thoracotomy. ${ }^{1}$ Adoption of minimally

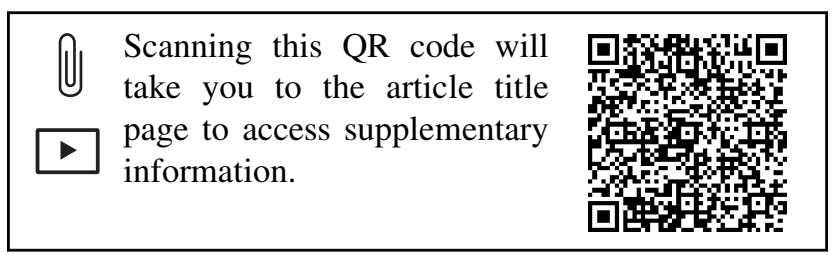




\section{Abbreviations and Acronyms \\ ASA = American Society of Anesthesiologists \\ $\mathrm{CI}=$ confidence interval \\ IPTW $=$ inverse probability of treatment weighting \\ STS = Society of Thoracic Surgery \\ VATS $=$ video-assisted thoracoscopic surgery}

invasive lobectomy by video-assisted thoracoscopic surgery (VATS) has been gradually increasing and more recently propelled after the introduction robotic-assisted thoracoscopic (robotic) lobectomy. ${ }^{2}$

With multiple established techniques, and little existing randomized data, controversy remains about the best approach. Although it is widely accepted that minimally invasive lobectomy is associated with improved perioperative outcomes, the utility of the robotics remains most controversial. $^{3-6}$ Among surgeons who have mastered the VATS approach, the robotic technology can be viewed as unnecessary and expensive equipment. ${ }^{7,8}$ Other surgeons have embraced robotics due to the improved optics, the full control of the operating surgeon, and the use of precise wristed instruments with increased freedom of motion..$^{9,10}$

In the current era of bundled payments and value-based care, surgeons are faced with increasing scrutiny to perform procedures associated with the best possible outcomes relative to dollars spent, ${ }^{11}$ and hospitals are carefully considering investments in expensive technology. ${ }^{12}$ Previous head-to-head comparisons of lobectomy approaches, including those of the early robotic experience, have indicated notable differences in cost. ${ }^{13-16}$ However, since the experience with robotic lung surgery has since evolved, these data may not accurately reflect the current outcomes and associated cost at high-volume centers. At our institution, we have gained substantial experience in robotic lung surgery and have continued to use VATS and open techniques to pulmonary lobectomy per preference of the individual surgeons. We hypothesize, that based on our current outcomes, there are no differences in overall cost to the hospital for robotic, VATS, and open lobectomy.

In this study, we sought to evaluate the hospital cost and outcomes of pulmonary lobectomy by robotic, VATS, and open thoracotomy approach performed and aim to overcome inherent differences in patient selection by statistical means of a propensity score weighted analysis.

\section{METHODS}

Patients who underwent pulmonary lobectomy between January 1, 2012, and September 1, 2017, were identified from the prospectively maintained institutional Society of Thoracic Surgery (STS) General Thoracic Surgery Database. We included all patients older than the age of 18 years with available financial data. Patients undergoing elective lobectomy for cancer or benign disease were included. We excluded patients who underwent sublobar resection, bronchoplastic resection, bilobectomy, or completion pneumonectomy. Pancoast tumors or concomitant chest wall resections were also excluded. Demographics, clinical, and outcomes data were extracted from the STS-General Thoracic Surgery Database. Definition of patient risk factors and postoperative events was defined by the STS Version 2.3 training manual. ${ }^{17}$ Major complications were assessed as the incidence of any major adverse outcome as previously described. ${ }^{18}$ Operative time was assessed as time from procedure start, typically a bronchoscopy, to completion of the operation following skin closure. Clinical data were then linked to hospital financial data provided by the billing department. The study was approved by our institutional review board with waiver of individual patient consent.

\section{Comparison Groups}

Patients were grouped by operative approach, distinguishing (1) robotic, (2) VATS, and (3) open thoracotomy. Selection of surgical approach was at the discretion of the treating surgeon. All procedures were performed by a group of board-certified thoracic surgeons and involved training surgical residents and cardiothoracic fellows. The experience level with minimally invasive techniques varied between the individual surgeons and evolved during the study period. The group included 3 high-volume minimally invasive surgeons, 1 primarily robotics surgeon, and 2 surgeons who have transitioned from VATS to robotics as the preferred approach for lobectomy within the study period. Although most surgeons have contributed to robotic, VATS, and open lobectomies in this cohort, 2 surgeons primarily performed open lobectomies.

Robotic lobectomy was performed using 4 instrument ports and an additional port for the bedside assistant. The da Vinci Si robot was initially used and replaced by the Xi model (Intuitive Surgical, Sunnyvale, Calif) in 2016. The majority of VATS cases were performed using a port-based approach with 4 ports, or a 3 -incision McKenna technique. ${ }^{19}$ Manual stapling was used in early robotic and all VATS and open lobectomies. Since 2016, we have also used the EndoWrist controlled staplers (Intuitive Surgical) for robotic lobectomies. Postoperative management was not protocolized but was guided by universal postoperative goals, including extubation in the operative room, recovery on a dedicated thoracic nursing wards, and chest tube removal before discharge, as previously described. ${ }^{20}$

\section{Measures of Cost and Clinical Effectiveness}

The primary financial outcome was total direct hospital cost. Secondary cost outcomes were total indirect cost specific to our institution and charges to the payor. Costs reflect the sum of all expenses to the hospital for the index stay for lobectomy, as provided by the billing department in US dollars. Both fixed and variable costs were included. Costs were determined at the discharge level and do not include outpatient expenses inquired for preoperative of postoperative care or readmissions. Total direct costs are defined as cost traceable to specific cost objects in the care of patients, including the price for any item used, facility occupied (operating room, hospital bed, etc), and service provided to conduct the operation and throughout the hospital stay. Total indirect costs are the sum of all additional costs for the hospital that is comprised of overhead cost and amortization of capital equipment, including of the purchase and maintenance of minimally invasive platforms, as previously outlined. ${ }^{21}$ Operating room cost as a fraction of total direct hospital cost was available for years 2012 to 2015. After 2015 , cost data were available on a more granular level by cost centers, which were compared separately. Charges were defined as the total dollar amount billed to the payor for the index hospitalization. Clinical effectiveness was measured by perioperative outcomes, taking into account length of stay, complications, and 30-day mortality.

\section{Statistical Analysis}

Categorical variables were summarized as frequencies and percentages. Crude comparisons were made between surgery groups using $\chi^{2}$ tests or 
TABLE 1. Patient characteristics and preoperative history in unadjusted and IPTW adjusted

\begin{tabular}{|c|c|c|c|c|c|c|c|c|}
\hline & \multicolumn{4}{|c|}{ Without IPTW, n (\%) } & \multicolumn{4}{|c|}{ With IPTW, \% } \\
\hline & $\begin{array}{l}\text { Robotic } \\
(\mathrm{n}=296)\end{array}$ & $\begin{array}{c}\text { VATS } \\
(n=161)\end{array}$ & $\begin{array}{c}\text { Open } \\
(n=240)\end{array}$ & $P$ value & $\begin{array}{l}\text { Robotic } \\
(\mathrm{n}=296)\end{array}$ & $\begin{array}{c}\text { VATS } \\
(n=160)\end{array}$ & $\begin{array}{c}\text { Open } \\
(n=240)\end{array}$ & $P$ value \\
\hline Age, y, mean $(\mathrm{SD}) /(95 \% \mathrm{CI})$ & $65.2(11.2)$ & $62.0(10.4)$ & $63.1(10.6)$ & .006 & $63.5(62.0-65.0)$ & $62.2(60.1-64.4)$ & $63.8(61.8-65.8)$ & .53 \\
\hline \multicolumn{9}{|l|}{ Sex } \\
\hline Female & $161(54.4)$ & $86(53.4)$ & $116(48.3)$ & .35 & $43 \%$ & $48 \%$ & $50 \%$ & .37 \\
\hline Male & $135(45.6)$ & $75(46.6)$ & $124(51.7)$ & & $57 \%$ & $52 \%$ & $50 \%$ & \\
\hline \multicolumn{9}{|l|}{ Race } \\
\hline White & $267(90.5)$ & $143(90.5)$ & $211(88.3)$ & .81 & $90 \%$ & $92 \%$ & $89 \%$ & .70 \\
\hline Black & $19(6.4)$ & $12(7.6)$ & $21(8.8)$ & & $7 \%$ & $7 \%$ & $7 \%$ & \\
\hline Asian/other & $9(3.1)$ & $3(1.9)$ & $7(2.9)$ & & $3 \%$ & $1 \%$ & $3 \%$ & \\
\hline BMI, mean $(\mathrm{SD}) /(95 \% \mathrm{CI})$ & $27.9(6.7)$ & $27.8(6.1)$ & $28.1(6.9)$ & .93 & $27.9(27.0-28.7)$ & $27.7(26.5-28.8)$ & $28.0(27.2-28.9)$ & .88 \\
\hline \multicolumn{9}{|l|}{ Primary payor } \\
\hline $\begin{array}{l}\text { Medicare/Medicaid/state/ } \\
\text { military }\end{array}$ & $107(36.1)$ & $88(54.7)$ & $97(40.4)$ & $<.001$ & $40 \%$ & $44 \%$ & $38 \%$ & .91 \\
\hline HMO/commercial & $178(60.1)$ & $61(37.9)$ & $134(55.8)$ & & $55 \%$ & $51 \%$ & $56 \%$ & \\
\hline Self/none & $11(3.7)$ & $12(7.5)$ & $9(3.8)$ & & $4 \%$ & $5 \%$ & $5 \%$ & \\
\hline \multicolumn{9}{|l|}{ Smoking status } \\
\hline Never & $58(19.6)$ & $36(22.5)$ & $45(18.8)$ & .79 & $18 \%$ & $21 \%$ & $19 \%$ & .53 \\
\hline Former & $141(47.6)$ & $78(48.8)$ & $123(51.2)$ & & $47 \%$ & $54 \%$ & $48 \%$ & \\
\hline Active & $97(32.8)$ & $46(28.8)$ & $72(30.0)$ & & $34 \%$ & $25 \%$ & $33 \%$ & \\
\hline $\begin{array}{l}\text { Pack-years of cigarette use, } \\
\text { mean }(\mathrm{SD}) /(95 \% \mathrm{CI})\end{array}$ & $43.6(27.0)$ & $42.0(26.3)$ & $45.2(30.2)$ & .62 & $42.3(38.5-46.2)$ & $44.9(38.3-51.6)$ & $43.8(39.7-48.0)$ & .76 \\
\hline \multicolumn{9}{|l|}{ Zubrod score } \\
\hline $0-1$ & 271 (91.6) & $151(93.8)$ & $220(91.7)$ & .67 & $92 \%$ & $92 \%$ & $94 \%$ & .69 \\
\hline $2+$ & $25(8.4)$ & $10(6.2)$ & $20(8.3)$ & & $8 \%$ & $8 \%$ & $6 \%$ & \\
\hline \multicolumn{9}{|l|}{ ASA score } \\
\hline 2 & $11(3.7)$ & $12(7.5)$ & $6(2.5)$ & .02 & $5 \%$ & $5 \%$ & $4 \%$ & $>.99$ \\
\hline 3 & $238(80.4)$ & $136(84.5)$ & $193(80.4)$ & & $81 \%$ & $82 \%$ & $82 \%$ & \\
\hline 4 & 47 (15.9) & $13(8.1)$ & $41(17.1)$ & & $14 \%$ & $14 \%$ & $14 \%$ & \\
\hline Hypertension & $190(64.2)$ & $88(54.7)$ & $144(60.0)$ & .13 & $60 \%$ & $58 \%$ & $60 \%$ & .92 \\
\hline Diabetes mellitus & $28(9.5)$ & $25(15.5)$ & $29(12.1)$ & .15 & $11 \%$ & $14 \%$ & $11 \%$ & .76 \\
\hline Congestive heart failure & $1(0.3)$ & $1(0.6)$ & $1(0.4)$ & $>.99$ & $0 \%$ & $5 \%$ & $0 \%$ & $<.01$ \\
\hline Coronary artery disease & $123(41.6)$ & $38(23.6)$ & $105(43.8)$ & $<.001$ & $38 \%$ & $31 \%$ & $39 \%$ & .41 \\
\hline Peripheral vascular disease & $30(10.1)$ & $5(3.1)$ & $14(5.8)$ & .01 & $6 \%$ & $2 \%$ & $7 \%$ & .18 \\
\hline $\begin{array}{l}\text { Previous cardiothoracic } \\
\text { surgery }\end{array}$ & $43(14.5)$ & $17(10.6)$ & $50(20.8)$ & .02 & $14 \%$ & $17 \%$ & $15 \%$ & .82 \\
\hline Preoperative chemotherapy & $11(3.7)$ & $11(6.8)$ & $25(10.4)$ & .009 & $7 \%$ & $7 \%$ & $7 \%$ & $>.99$ \\
\hline $\begin{array}{l}\text { Preoperative thoracic } \\
\text { radiation }\end{array}$ & $12(4.1)$ & $9(5.6)$ & $26(10.8)$ & .006 & $7 \%$ & $7 \%$ & $7 \%$ & $>.99$ \\
\hline Cerebrovascular accident & $9(3.0)$ & $4(2.5)$ & $14(5.8)$ & .15 & $4 \%$ & $3 \%$ & $4 \%$ & .93 \\
\hline Steroids & $9(3.0)$ & $3(1.9)$ & $9(3.8)$ & .56 & $4 \%$ & $6 \%$ & $2 \%$ & .64 \\
\hline $\begin{array}{l}\text { Last creatinine level, } \mathrm{mg} / \mathrm{dL} \text {, } \\
\text { mean }(\mathrm{SD}) /(95 \% \mathrm{CI})\end{array}$ & $0.9(0.3)$ & $0.9(0.3)$ & $0.9(0.5)$ & .44 & $0.9(0.8-0.9)$ & $0.9(0.9-1.0)$ & $0.9(0.8-0.9)$ & .86 \\
\hline $\begin{array}{l}\text { Last hemoglobin level, g/dL, } \\
\text { mean }(\mathrm{SD}) /(95 \% \mathrm{CI})\end{array}$ & $13.6(1.6)$ & $13.6(1.6)$ & 12.9 (1.9) & $<.001$ & $13.4(13.2-13.6)$ & $13.5(13.0-14.0)$ & $13.4(13.1-13.6)$ & .89 \\
\hline COPD & $133(44.9)$ & $55(34.2)$ & $97(40.4)$ & .08 & $41 \%$ & $42 \%$ & $44 \%$ & .81 \\
\hline $\begin{array}{l}\% \text { FEV1 mean }(\mathrm{SD}) / \\
(95 \% \mathrm{CI})\end{array}$ & $82.3(20.3)$ & $85.3(19.0)$ & $77.8(20.1)$ & .001 & $81.3(78.8-83.8)$ & $78.3(71.2-85.4)$ & $82.5(79.1-85.9)$ & .57 \\
\hline
\end{tabular}




\begin{tabular}{|c|c|c|c|c|c|c|c|c|}
\hline & \multicolumn{4}{|c|}{ Without IPTW, n (\%) } & \multicolumn{4}{|c|}{ With IPTW, \% } \\
\hline & $\begin{array}{c}\text { Robotic } \\
(n=296)\end{array}$ & $\begin{array}{c}\text { VATS } \\
(n=161)\end{array}$ & $\begin{array}{c}\text { Open } \\
(n=240)\end{array}$ & $P$ value & $\begin{array}{c}\text { Robotic } \\
(n=296)\end{array}$ & $\begin{array}{c}\text { VATS } \\
(\mathbf{n}=160)\end{array}$ & $\begin{array}{c}\text { Open } \\
(n=240)\end{array}$ & $P$ value \\
\hline $\begin{array}{l}\% \text { DLCO mean }(\mathrm{SD}) / \\
\quad(95 \% \mathrm{CI})\end{array}$ & $76.1(22.7)$ & $86.9(22.0)$ & $73.3(20.3)$ & $<.001$ & $77.4(74.3-80.6)$ & $77.8(70.3-85.2)$ & $77.3(74.1-80.5)$ & .99 \\
\hline Lung cancer & $252(85.1)$ & $144(89.4)$ & $196(81.7)$ & .10 & $85 \%$ & $79 \%$ & $85 \%$ & .38 \\
\hline \multicolumn{9}{|l|}{ Clinical T stage } \\
\hline No cancer & $44(14.86)$ & $17(10.63)$ & $44(18.33)$ & $<.001$ & $15 \%$ & $21 \%$ & $15 \%$ & .90 \\
\hline cT1 & $155(52.36)$ & $89(55.63)$ & $87(36.25)$ & & $47 \%$ & $43 \%$ & $47 \%$ & \\
\hline cT2 & $46(15.54)$ & $38(23.75)$ & $43(17.92)$ & & $19 \%$ & $20 \%$ & $19 \%$ & \\
\hline cT3 & $29(9.80)$ & $7(4.38)$ & $30(12.50)$ & & $11 \%$ & $5 \%$ & $9 \%$ & \\
\hline cT4 & $15(5.07)$ & $8(5.00)$ & $27(11.25)$ & & $6 \%$ & $11 \%$ & $8 \%$ & \\
\hline Unknown & $7(2.36)$ & $1(0.63)$ & $9(3.75)$ & & $2 \%$ & $1 \%$ & $2 \%$ & \\
\hline \multicolumn{9}{|l|}{ Clinical N stage } \\
\hline No cancer & $44(14.86)$ & $17(10.63)$ & $44(18.33)$ & .010 & $15 \%$ & $21 \%$ & $15 \%$ & .86 \\
\hline cNO & $227(76.69)$ & $121(75.63)$ & $153(63.75)$ & & $71 \%$ & $67 \%$ & $71 \%$ & \\
\hline $\mathrm{cN} 1$ & $11(3.72)$ & $10(6.25)$ & $21(8.75)$ & & $6 \%$ & $5 \%$ & $6 \%$ & \\
\hline $\mathrm{cN} 2$ & $14(4.73)$ & $12(7.50)$ & $22(9.17)$ & & $8 \%$ & $6 \%$ & $8 \%$ & \\
\hline \multicolumn{9}{|l|}{ Lobe } \\
\hline Upper lobe & $144(48.65)$ & $88(55.00)$ & 154 (64.17) & .030 & $55 \%$ & $51 \%$ & $57 \%$ & .86 \\
\hline Middle lobe & $23(7.77)$ & $11(6.88)$ & $14(5.83)$ & & $7 \%$ & $7 \%$ & $7 \%$ & \\
\hline Lower lobe & $120(40.54)$ & $56(35.00)$ & 64 (26.67) & & $35 \%$ & $36 \%$ & $33 \%$ & \\
\hline Unknown & $9(3.04)$ & $5(3.13)$ & $8(3.33)$ & & $3 \%$ & $6 \%$ & $3 \%$ & \\
\hline
\end{tabular}

Values in bold indicate statistically significant $P$ values. IPTW, Inverse probability treatment weight; Robotic, robotic-assisted thoracoscopic surgery; VATS, video-assisted thoracoscopic surgery; Open, open thoracotomy approach; $S D$, standard deviation; $C I$, confidence interval; $B M I$, body mass index; $H M O$, health maintenance organization; $A S A$, American Society of Anesthesiology; $C O P D$, chronic obstructive pulmonary disease; $F E V I$, forced expiratory volume in 1 second; $D L C O$, diffusing capacity of carbon monoxide.

Fisher exact tests where relevant. Continuous baseline characteristics were reported as means (standard deviations) or medians [interquartile ranges] and analysis of variance was used to test for differences between surgery groups. To account for treatment selection bias, we attempted to balance the baseline characteristics and surgical risk factors between the 3 lobectomy groups using a propensity score methodology with inverse probability of treatment weighing (IPTW). ${ }^{22}$ The IPTWs were estimated using multinomial logistic regression analysis including the following differential variables $(P \leq .15)$, and disregarding outcomes: age, primary payor, American Society of Anesthesiologists (ASA) score, hypertension, diabetes, coronary artery disease, peripheral vascular disease, preoperative chemotherapy, preoperative thoracic radiation, prior cardiothoracic surgery, history of cerebrovascular accident, chronic obstructive lung disease, preoperative forced expiratory volume in 1 second, diffusion capacity for carbon monoxide, last preoperative hemoglobin and creatinine, presence of cancer, clinical tumor and nodal stage, and lobe resected. Complete data for these variables were available for $87.7 \%$ of patients. Data were assumed to be missing at random.

Multivariate normal imputation using SAS Proc MI was carried out to impute 10 data sets with imputed values for missing baseline characteristics that were then used to calculate the IPTWs for each patient. Inverse probability of treatment weights was calculated for each patient in each of the imputed data sets. The mean stabilized weights for each patient from the 10 data sets were used as the final IPTWs. Patients with overweighted IPTWs (scores $>15$ ) were excluded from the adjusted analyses, which was the case for 1 patient in the VATS group. Patient characteristics were then reassessed for balance between surgery groups after adjusting for the IPTWs.

Baseline categorical variables and perioperative outcomes were compared between surgery groups after adjusting for IPTWs using RaoScott $\chi^{2}$ tests using the Surveyfreq procedure in SAS. The SAS Surveyreg procedure was used to fit IPTW adjusted linear regression models to test for differences in continuous variables between surgery groups for each outcome respectively. For pairwise comparison of cost endpoints, multiple comparisons of cohort differences were adjusted for using the TukeyKramer method. Cost outcomes were assessed on the natural log scale due to violations of the normality assumption on the original scale. To test the impact of conversions of minimally invasive to open procedures, we first excluded conversions in the cost analysis, and then repeated the analysis accounted for conversions in an intention-to-treat analysis. Hypothesis testing for main outcomes was conducted maintaining an overall $5 \%$ type I error rate. The statistical analysis was designed and conducted by a biostatistician (M.A.) using SAS version 9.4 (SAS Institute, Cary, NC).

\section{RESULTS \\ Patient Characteristics}

A total of 697 patients who fulfilled the selection criteria underwent lobectomy completed by robotic $(\mathrm{n}=296)$ and VATS $(n=161)$ or open thoracotomy $(n=240)$ approach. Forty-one patients were converted from robotic approach to thoracotomy and 21 from VATS to thoracotomy. A Consolidated Standards of Reporting Trials diagram detailing the patient selection is provided as Figure E1. Patient demographics and clinical characteristics before and after propensity weighing are summarized in Table 1. Before the propensity weighing, the robotic, VATS, and open cohorts differed in multiple variables, including age, insurance/payor status, ASA score, comorbidities, pulmonary function test, resection following previous 
thoracic surgery or neoadjuvant therapy, and type of lobe resected.

Although risk factors were similar between robotic and open thoracotomy patients, patients who underwent VATS on average were younger and had lower ASA scores, fewer cardiovascular comorbidities, and greater preoperative forced expiratory volume in 1 second and diffusion capacity for carbon monoxide (Table 1). The thoracotomy group had the greatest rate of upper lobes resections (robotic $50 \%$ vs VATS $57 \%$ vs open $66 \%, P=.008$ ), subset of patients with previous chest surgery (robotic $15 \%$ vs VATS $11 \%$ vs open $21 \%, P=.02$ ), as well as subset of patients treated with neoadjuvant chemoradiotherapy therapy (robotic $4 \%$ vs
VATS $6 \%$ vs open $11 \%, P=.006)$. After IPTW adjustment, similar distribution of covariates was achieved among the robotic, VATS, and open thoracotomy groups in the weighted cohorts (Table 1).

\section{Perioperative Outcomes}

A comparison of crude and IPTW-adjusted perioperative outcomes by approach is presented in Table 2 . The unadjusted rate of any complication (robotic $46 \%$ vs VATS $39 \%$ vs open $56 \%, P=.002$ ) and major complication (robotic $12 \%$ vs VATS $6 \%$ vs open $16 \%, P=.006$ ) were lowest in the VATS group and greatest in the open thoracotomy group. After IPTW adjustment, these differences were

TABLE 2. Perioperative characteristics before and after IPTW adjustment

\begin{tabular}{|c|c|c|c|c|c|c|c|c|}
\hline & \multicolumn{4}{|c|}{ Without IPTW, n (\%) } & \multicolumn{4}{|c|}{ With IPTW, \% } \\
\hline & Robotic & VATS & Open & $P$ value & Robotic & VATS & Open & $P$ value \\
\hline LOS, median [IQR] & $4[3,6]$ & $4[3,6]$ & $6[5,9]$ & $<.001$ & $3.8[2.6,5.9]$ & $3.8[2.8,7.0]$ & $5.4[4.0,7.6]$ & $<.001$ \\
\hline $\begin{array}{l}\text { Operating room time, } \\
\text { min, mean }(\mathrm{SD}) / \\
(95 \% \mathrm{CI})\end{array}$ & $283.6(71.9)$ & $313.2(74.6)$ & $287.5(92.5)$ & $<.001$ & $287.2(278.2-296.2)$ & $305.8(288.7-323.0)$ & 278.9 (265.1-292.8) & .05 \\
\hline Any complication & $136(45.9)$ & $62(38.5)$ & $134(55.8)$ & .002 & $45 \%$ & $45 \%$ & $55 \%$ & .16 \\
\hline Major complications & $35(11.82)$ & $9(5.63)$ & $39(16.25)$ & .006 & $12 \%$ & $12 \%$ & $15 \%$ & .70 \\
\hline Pulmonary complications & $64(22.8)$ & $30(20.1)$ & 77 (33.6) & .004 & $21 \%$ & $31 \%$ & $31 \%$ & .15 \\
\hline Air leak & $16(5.4)$ & $14(8.7)$ & $26(10.8)$ & .06 & $5 \%$ & $9 \%$ & $9 \%$ & .32 \\
\hline Atelectasis & $17(5.7)$ & $5(3.1)$ & $38(15.8)$ & $<.001$ & $5 \%$ & $6 \%$ & $16 \%$ & .03 \\
\hline $\begin{array}{l}\text { Pleural effusion } \\
\text { requiring drainage }\end{array}$ & $5(1.7)$ & $3(1.9)$ & $6(2.5)$ & .79 & $2 \%$ & $3 \%$ & $2 \%$ & .88 \\
\hline Pneumonia & $10(3.4)$ & $6(3.7)$ & $17(7.1)$ & .13 & $3 \%$ & $2 \%$ & $8 \%$ & $<.01$ \\
\hline ARDS & $0(0.0)$ & $0(0.0)$ & $5(2.1)$ & .005 & $0 \%$ & $0 \%$ & $1 \%$ & $\mathrm{n} / \mathrm{a}$ \\
\hline Respiratory failure & $22(7.4)$ & $5(3.1)$ & $27(11.3)$ & .009 & $8 \%$ & $9 \%$ & $8 \%$ & .98 \\
\hline Pneumothorax & $13(4.4)$ & $3(1.9)$ & $7(2.9)$ & .34 & $4 \%$ & $1 \%$ & $2 \%$ & .13 \\
\hline $\begin{array}{l}\text { Cardiovascular } \\
\text { complications }\end{array}$ & $32(10.8)$ & $16(9.9)$ & $40(16.7)$ & .07 & $10 \%$ & $13 \%$ & $15 \%$ & .52 \\
\hline Atrial fibrillation & $24(8.1)$ & $14(8.7)$ & $27(11.3)$ & .45 & $8 \%$ & $7 \%$ & $10 \%$ & .57 \\
\hline Infectious complications & $17(6.4)$ & $8(5.6)$ & $23(10.0)$ & .22 & $6 \%$ & $10 \%$ & $9 \%$ & .58 \\
\hline Surgical-site infection & $11(3.7)$ & $4(2.5)$ & $6(2.5)$ & .72 & $3 \%$ & $3 \%$ & $2 \%$ & .70 \\
\hline $\begin{array}{l}\text { Gastrointestinal } \\
\text { complications }\end{array}$ & $7(2.4)$ & $4(2.5)$ & $9(3.8)$ & .62 & $2 \%$ & $2 \%$ & $4 \%$ & .40 \\
\hline Ileus & $5(1.7)$ & $2(1.2)$ & $4(1.7)$ & $>.99$ & $2 \%$ & $1 \%$ & $2 \%$ & .82 \\
\hline Genitourinary complications & $54(18.2)$ & $22(13.7)$ & $39(16.3)$ & .45 & $17 \%$ & $16 \%$ & $15 \%$ & .93 \\
\hline Renal failure & $5(1.7)$ & $2(1.2)$ & $9(3.8)$ & .21 & $2 \%$ & $6 \%$ & $3 \%$ & .36 \\
\hline Postoperative transfusion & $11(3.7)$ & $7(4.3)$ & $34(14.2)$ & $<.001$ & $4 \%$ & $14 \%$ & $10 \%$ & .06 \\
\hline Return to operating room & $4(1.4)$ & $2(1.2)$ & $7(2.9)$ & .37 & $1 \%$ & $1 \%$ & $4 \%$ & .13 \\
\hline Discharge with chest tube & $16(5.4)$ & $8(5.0)$ & $23(9.6)$ & .12 & $5 \%$ & $9 \%$ & $7 \%$ & .57 \\
\hline ICU stay & $31(10.5)$ & $5(3.1)$ & 35 (14.6) & $<.001$ & $10 \%$ & $9 \%$ & $11 \%$ & .94 \\
\hline $\begin{array}{l}\text { ICU days* (mean (SD)/ } \\
\quad(95 \% \mathrm{CI})\end{array}$ & $4.4(1.2)$ & $6.0(1.6)$ & $7.4(1.2)$ & .15 & $3.9(2.8-5.4)$ & $5.0(3.9-6.5)$ & $6.8(4.3-10.8)$ & .13 \\
\hline Mortality (30 d) & $3(1.0)$ & $1(0.6)$ & $4(1.7)$ & .73 & $1 \%$ & $5 \%$ & $2 \%$ & .12 \\
\hline
\end{tabular}

Values in bold indicate statistically significant $P$ values. IPTW, Inverse probability treatment weight; Robotic, robotic-assisted thoracoscopic surgery; VATS, video-assisted thoracoscopic surgery; Open, open thoracotomy approach; $L O S$, length of stay; $I Q R$, interquartile range; $S D$, standard deviation; $C I$, confidence interval; $I C U$, intensive care unit; $A R D S$, acute respiratory distress syndrome; $n / a$, not applicable. *ICU days compared for patients admitted to the ICU only. 
TABLE 3. Propensity score-adjusted cost comparison by surgical approach*

\begin{tabular}{|c|c|c|c|c|c|c|}
\hline \multirow[b]{2}{*}{ Cost } & \multicolumn{3}{|c|}{ Mean cost $(95 \%$ CI $)$, US \$ } & \multicolumn{3}{|c|}{$P$ value } \\
\hline & Robotic $(N=296)$ & $\operatorname{VATS}(\mathbf{N}=160)$ & Open $(N=240)$ & $\begin{array}{l}\text { Robotic vs } \\
\text { VATS }\end{array}$ & $\begin{array}{l}\text { Open vs } \\
\text { robotic }\end{array}$ & $\begin{array}{l}\text { Open vs } \\
\text { VATS }\end{array}$ \\
\hline Total direct costs $\dagger$ & $\begin{array}{c}17,222.8 \\
(16,405.3-18,081.0)\end{array}$ & $\begin{array}{c}17,259.7 \\
(15,982.5-18,639.0)\end{array}$ & $\begin{array}{c}18,074.6 \\
(16,920.4-19,307.6)\end{array}$ & $>.99$ & .48 & .64 \\
\hline $\begin{array}{l}\text { Total indirect } \\
\text { costs } \dagger\end{array}$ & $\begin{array}{c}17,214.9 \\
(16,437.4-18,029.2)\end{array}$ & $\begin{array}{c}16,414.5 \\
(15,191.0-17,736.5)\end{array}$ & $\begin{array}{c}16,993.4 \\
(15,941.4-18,114.9)\end{array}$ & .55 & .94 & .78 \\
\hline $\begin{array}{l}\text { Operating room } \\
\text { costs } \ddagger\end{array}$ & $\begin{array}{c}9,911.8 \\
(9531.1-10,292.5)\end{array}$ & $\begin{array}{c}9,491.2 \\
(8936.6-10,045.9)\end{array}$ & $\begin{array}{c}8,697.8 \\
(8177.9-9217.7)\end{array}$ & .44 & $<.01$ & .10 \\
\hline Total charges $\dagger$ & $\begin{array}{c}119,179.6 \\
(114,399.2-124,159.8)\end{array}$ & $\begin{array}{c}124,026.0 \\
(115,458.5-133,229.3)\end{array}$ & $\begin{array}{c}120,811.3 \\
(113,464.3-128,633.9)\end{array}$ & .61 & .93 & .85 \\
\hline
\end{tabular}

Values in bold indicate statistically significant $P$ values. $C I$, Confidence interval; Robotic, robotic-assisted thoracoscopic surgery; VATS, video-assisted thoracoscopic surgery; Open, open thoracotomy approach. *Multiple comparisons of cohort differences were adjusted for using the Tukey-Kramer method. $\dagger$ Geometric mean and $95 \%$ confidence interval $(95 \% \mathrm{CI})$ based on $\log$ scale values transformed back to costs expressed as US dollar. †Operating room costs data were only available from 2012 to 2015,518 cased are included (robotic $=227$, VATS $=84$ and open $=207$ ).

less pronounced (Table 2). Increased rates of atelectasis requiring bronchoscopy (robotic $5 \%$ vs VATS $6 \%$ vs open $16 \%, P=.03$ ) and pneumonia (robotic $3 \%$ vs VATS $2 \%$ vs open $8 \%, P=.01$ ) remained significantly greater in patients approached by thoracotomy and were identical between robotic and VATS lobectomy patients. The IPTW-adjusted mean operative times were lowest for open thoracotomy and longest for a VATS lobectomy (robotic 278 minutes vs VATS 289 minutes vs open 265 minutes, $P=.05$ ). Median length of hospital stay was similar between the patients undergoing robotic-assisted and VATS and significantly longer following open lobectomy (robotic 3.8 days vs VATS 3.8 days vs open 5.4 days, $P<.001$ ).

\section{Cost Comparison}

A propensity-adjusted cost comparison by surgical approach is presented in Table 3, and the unadjusted analysis is provided in Table E1. The IPTW-adjusted mean total direct hospital cost for robotic lobectomy was $\$ 17,223$ ( $95 \%$ confidence interval $[\mathrm{CI}], \$ 16,405-\$ 18,081)$ and was not significantly different as compared with VATS lobectomy $(\$ 17,259$ [95\% CI: $\$ 15,983-\$ 18,639] ; P>.99)$, or open lobectomy $(\$ 18,075$ [95\% CI: $16,920-19,308$; $P=.48)$. The distribution and variability of total direct cost are illustrated in box plot graphs in the supplement (Figure E2). There was also no difference in total indirect cost (Table 3).

Cost comparisons remained unchanged when considering conversions of minimally invasive procedures, assessed by an intention-to-treat analysis (Table E2). Operating room cost of robotic lobectomy was significantly greater than for open but similar to VATS lobectomy. A detailed breakdown of proportional costs by the various cost centers is compared in Table 4. We observed an inverse relationship between operating room cost and the cost for the hospital room and nursing among the 3 groups. Robotic

TABLE 4. Total direct cost breakdown analysis

\begin{tabular}{|c|c|c|c|c|}
\hline$\%$ of total direct cost, mean (SD) & $\begin{array}{c}\text { Robotic } \\
(N=69)\end{array}$ & $\begin{array}{c}\text { VATS } \\
(\mathbf{N}=76)\end{array}$ & $\begin{array}{c}\text { Open } \\
(\mathbf{N}=\mathbf{3 3})\end{array}$ & $P$ value \\
\hline Preoperation cost & $2.3(1.3)$ & $3.1(2.1)$ & $2.7(1.4)$ & .01 \\
\hline OR/ASU/PACU cost & $48.5(14.9)$ & $47.5(9.6)$ & $42.2(12.3)$ & .05 \\
\hline Anesthesia cost & $3.2(1.0)$ & $4.2(1.0)$ & $3.9(1.2)$ & $<.001$ \\
\hline ICU cost & $1.2(4.9)$ & $0.0(0.0)$ & $1.5(8.9)$ & .78 \\
\hline Ward/nursing cost & $15.2(6.8)$ & $19.5(8.6)$ & $22.7(9.6)$ & $<.001$ \\
\hline Pharmacy/Blood Bank/central supply cost & $7.7(4.2)$ & $7.3(3.3)$ & $8.5(4.5)$ & .31 \\
\hline Laboratory/path cost & $15.0(17.5)$ & $11.7(7.0)$ & $9.6(8.7)$ & .09 \\
\hline Imaging/tests/IR cost & $3.2(1.3)$ & $3.3(1.2)$ & $4.3(1.3)$ & $<.001$ \\
\hline Consulting services cost & $0.4(2.7)$ & $0.6(1.7)$ & $0.1(0.2)$ & .47 \\
\hline PT/OT/SLP/RT cost & $3.2(2.0)$ & $2.7(2.5)$ & $4.4(2.4)$ & $<.001$ \\
\hline
\end{tabular}

Values in bold indicate statistically significant $P$ values. Robotic, Robotic-assisted thoracoscopic surgery; VATS, video-assisted thoracoscopic surgery; Open, open thoracotomy approach; $O R$, operating room; $A S U$, ambulatory surgery unit; $P A C U$, postoperative care unit; $I C U$, internsive care unit; $I R$, interventional radiology; $P T$, physical therapy; $O T$, occupational therapy; $S L P$, speech language pathology; $R T$, respiratory therapy. 


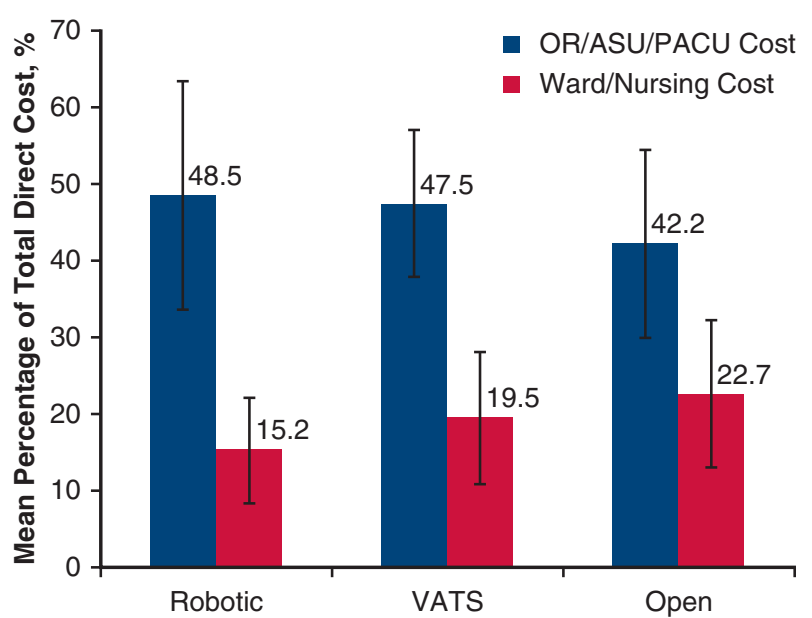

FIGURE 1. Proportion procedure-related cost (incuding OR, preoperative ASU, and PACU cost) relative to mean postoperative recovery cost (including hospital ward and nursing cost) as mean percentages of total direct hospital cost by operative approach to pulmonary lobectomy. Error bars represent standard deviations. Robotic, Robotic-assisted thoracoscopic surgery; VATS, video-assisted thoracoscopic surgery; Open, open thoracotomy approach; $O R$, operating room; $A S U$, ambulatory surgical unit; $P A C U$, postanesthesia care unit.

lobectomy was associated with the greatest operating room cost but lowest hospital room/nursing cost (Figure 1). The IPTW-adjusted mean charges to the payor did not differ among the robotic, VATS, and open lobectomy cohorts (robotic $\$ 119,180$ vs VATS $\$ 124,026$ vs open $\$ 120,811$; $P=.63)$.

\section{DISCUSSION}

The high cost of robotic surgery has been a main point of criticism since the early adoption of robotic-assisted lung resection and has raised the question of its value. In this retrospective study of a large single-institution experience, we demonstrate that there were no significant differences in hospital cost or charges to the payor among roboticassisted, VATS, and open lobectomies in a propensity weight-adjusted analysis. The clinical effectiveness of robotic lobectomy as measured by length of stay and postoperative events was similar to that of the VATS cases. The clinical outcomes of both minimally invasive procedures compared favorably with open thoracotomy, with reduced pulmonary complications and decreased length of stay, even after carefully controlling for patient selection (Graphical Abstract, Video 1). In a recent systematic review of published studies on cost of robotic lobectomy, we have shown that there is great variability among institutions, ranging between $\$ 15,440$ and $\$ 22,582$ for median hospital cost. ${ }^{21}$ We showed that the greatest hospital costs of robotic lobectomy were calculated from registry data, reflecting the early robotic experience. ${ }^{7,13}$ By contrast, the lowest costs associated with robotic lobectomy were reported from a

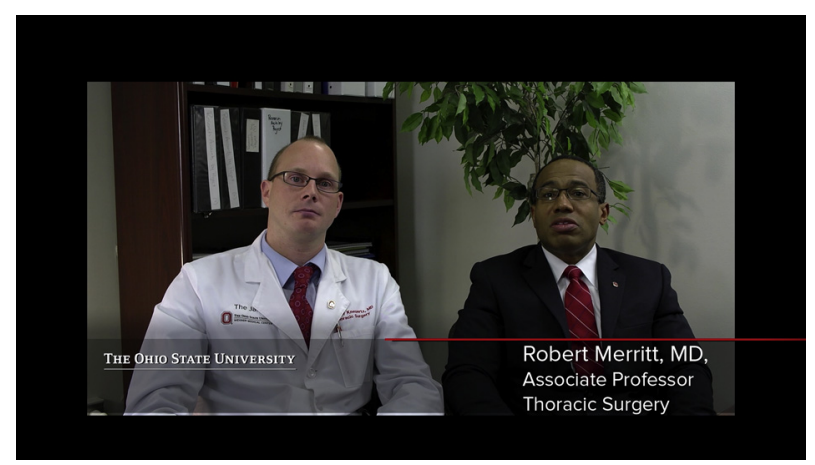

VIDEO 1. The first and senior authors giving a summary and discussing the implications of the study. Video available at: https://www.jtcvs.org/ article/S0022-5223(19)30064-9/fulltext.

high-volume center, which also reported the lowest complication rates and shortest hospital stay. ${ }^{15}$

A previous analysis of factors associated with cost of lobectomy showed that the additive cost of postoperative events had a substantial impact on overall hospital cost following lobectomy, which increased by $23 \%, 53 \%$, and $185 \%$ with 1,2 , and 3 complications, respectively. ${ }^{23} \mathrm{Im}-$ provements of postoperative outcomes therefore have a notable impact on overall cost-effectiveness. ${ }^{20}$ In one of the first cost-effectiveness studies, Burfeind and colleagues ${ }^{24}$ also reported on cost savings and at a single center with high volume of VATS. Swanson and others ${ }^{16}$ confirmed that increased operating room cost of VATS lobectomy can be mitigated by improved postoperative outcomes in a multi-institution analysis. Our current analysis confirms that procedural cost in the operating room remains lowest with open procedures, as it is associated with the shortest operating room times and the least expensive equipment. However, even after carefully adjusting for patient selection, both robotic and VATS approaches were associated with shorter length of stay and fewer costly events, and, as a result, in similar overall hospital cost. Robotic lobectomy cases had the greatest operating room costs, which were proportionally recovered by savings in the postoperative period (Figure 1).

Our results of cost comparison of robotic and VATS lobectomy differ from those of previous studies, which were derived from the early experience of the robotic lobectomy. ${ }^{7,13,14}$ Overall costs were greater than 2 large registry studies, which found that robotic lobectomy was more costly than VATS lobectomy, whereas there was no significant difference in this current study: Paul and colleagues ${ }^{13}$ compared the outcomes of the early experience of robotic to VATS lobectomy using the National Inpatient Sample and found greater complication rates with robotic lobectomy, with an estimated cost increase of $26 \%$. Similarly, Swanson and colleagues ${ }^{7}$ found a that cost of robotic lobectomy resulted in nearly $22 \%$ greater hospital cost than for 
VATS lobectomy in the Premier Database, which was associated with longer operating times for robotic procedures. These studies pooled cases from the early robotic experience (years 2008-2011). In a more recent, small single institution study, Deen and colleagues ${ }^{8}$ compared hospital costs of 57 robotic with 58 VATS lobectomies and showed that robotic cases were still more expensive, with greater costs derived from longer operative times and the added cost of roboticspecific supplies and depreciation. In the present study, we show that with shorter operative times and similar outcomes, robotic lobectomy can be performed with similar cost as compared with the VATS approach.

Looking beyond the perspective of hospital economics, it is evident that the choice of surgical approach may have a broader impact on health economics and the surgical community. A recent study by Watson and $\mathrm{Qiu}^{25}$ used a large commercial insurance claims database to examine differences of thoracoscopic and open lobectomies. Although their comparison was not adjusted for patient selection, it showed that the thoracoscopic approach was associated with less resource use. They demonstrated a $24 \%$ lower use of health care days within 90 days and 14\% less resource use in the first operative year, which points to potential cost savings beyond the index hospitalization. ${ }^{25}$ Robotic lobectomy cases were likely included in the thoracoscopic group, because there was no specific Current Procedural Terminology code. Although few studies to date have compared hospital charges to the payor, one previous study had concluded that total hospital charges of robotic lobectomy exceeded those of VATS lobectomy by $45 \%{ }^{13}$ in the early robotic experience, possibly due to the reported greater intraoperative and bleeding complication rates. In our current study, we show that with similar outcomes between robotic and VATS procedures, total hospital charges of robotic, VATS, and open lobectomy were similar in the adjusted analysis.

Finally, our data are perhaps most impactful from the perspective of the individual cost-considerate surgeon. Our results indicate that primary criterion of selection of the lobectomy approach should be driven by the surgeon's experience and comfort level. Although cost should remain a consideration, it should not dictate the surgical approach. This is by reason that in the overall in-hospital equation, postoperative outcomes appear to be the main determinant of cost and ultimately determine what benefits patients. As for many innovative procedures, the true value of robotic lobectomy may not be apparent at first. The upfront capital cost of the robotic technology remains high in the current single manufacturer market, and the clinical outcomes have not been proven to be significantly better compared with the VATS approach. Until the price of technology decreases, which is expected in the near future with the arrival new platforms of competing manufacturers, surgeons may need to continue to debate over the utility of using robotics. We recognize that the realization of economies of scale at our institution is likely the result of a system-wide commitment to robotic surgery and the work of a dedicated robotics team with continued process improvement efforts. As such, the operative time of robotic lobectomy has been reduced to an average duration shorter than that of VATS lobectomy, and recovery has been streamlined. With growing experience of robotic thoracic surgery, additional important benefits are now being recognized in the surgical community. This includes the steep learning curve of trainees, resulting in increased competency of minimally invasive lobectomy.

Particular strengths of this study include the large number of patients treated at a single institution with detailed clinical data and the use of propensity scores to adjust for differences in selection in the comparison of the 3 approaches to lobectomy. There are, however, several limitations that must be considered when interpreting our results. As a result of the observational study design, unmeasured confounders cannot be completely excluded and may have influenced the comparison between groups. With regard to outcomes and cost, we have made every effort to control for differences in patient factors and known STS risk factors. However, there may other salient patient or disease factors that were not accounted for and could have influenced the selection of the operative approach. The groups are not randomized, and therefore the biases and experiences of individual surgeons, as well as the degree of trainee involvement, could have influenced the study results. Regarding the cost endpoints, we have provided a detailed analysis at the hospital level. Cost of postdischarge health care use, including readmissions and rehabilitation services were not considered and may have additional implications from a health economic standpoint. Financial analysis may not be directly comparable with other institutions, because cost factors may vary significantly between hospital systems. For example, other institutions may face different capital deprivation and amortization of the robot based on the overall volume of robotic cases. Comparability of absolute hospital cost between studies is further challenged by different cost definitions and time periods between studies. As such, the absolute cost figures presented, may appear higher or lower in this study as compared with some previous reports. Indirect cost in particular may differ most, based on the institutional economic conditions and operative volume. Lastly, this study is limited to comparison of hospital outcome measures and does not account for differences in patient reported outcomes and oncologic endpoints.

\section{CONCLUSIONS}

There were no significant differences in hospital cost or charges between the robotic-assisted, VATS, and open lobectomy. Increased procedural cost of minimally invasive lobectomy can be recovered in the postoperative period, associated 
with improved postoperative outcomes and shorter hospital stay. Robotic lobectomy can be performed with similar cost and outcomes as VATS at a high-volume center.

\section{Conflicts of Interest}

Dr Merritt is a speaker for Surgical Intuitive Inc. All other authors have nothing to disclose with regard to commercial support.

\section{References}

1. Rajaram R, Mohanty S, Bentrem DJ, Pavey ES, Odell DD, Bharat A, et al Nationwide assessment of robotic lobectomy for non-small cell lung cancer. Ann Thorac Surg. 2017;103:1092-100.

2. Oh DS, Reddy RM, Gorrepati ML, Mehendale S, Reed MF. Robotic-assisted, video-assisted thoracoscopic and open lobectomy: propensity-matched analysis of recent premier data. Ann Thorac Surg. 2017;104:1733-40.

3. Cheng D, Downey RJ, Kernstine K, Stanbridge R, Shennib H, Wolf R, et al. Video-assisted thoracic surgery in lung cancer resection: a meta-analysis and systematic review of controlled trials. Innovations (Phila). 2007;2:261-92.

4. Moffatt SD, Mitchell JD, Whyte RI. Role of video-assisted thoracoscopic surgery and classic thoracotomy in lung cancer management. Curr Opin Pulm Med. 2002;8:281-6.

5. Emmert A, Straube C, Buentzel J, Roever C. Robotic versus thoracoscopic lung resection: a systematic review and meta-analysis. Medicine (Baltimore). 2017; 96:e7633.

6. Long H, Tan Q, Luo Q, Wang Z, Jiang G, Situ D, et al. Thoracoscopic surgery versus thoracotomy for lung cancer: short-term outcomes of a randomized trial. Ann Thorac Surg. 2018;105:386-92.

7. Swanson SJ, Miller DL, McKenna RJ Jr, Howington J, Marshall MB, Yoo AC, et al. Comparing robot-assisted thoracic surgical lobectomy with conventional video-assisted thoracic surgical lobectomy and wedge resection: results from a multihospital database (Premier). J Thorac Cardiovasc Surg. 2014;147:929-37.

8. Deen SA, Wilson JL, Wilshire CL, Vallières E, Farivar AS, Aye RW, et al. Defining the cost of care for lobectomy and segmentectomy: a comparison of open, video-assisted thoracoscopic, and robotic approaches. Ann Thorac Surg. 2014;97:1000-7

9. Lin J. Robotic lobectomy: revolution or evolution? J Thorac Dis. 2017;9:2876-80.

10. Kneuertz PJ, D'Souza DM, Moffatt-Bruce SD, Merritt RE. Robotic lobectomy has the greatest benefit in patients with marginal pulmonary function. J Cardiothorac Surg. 2018;13:56.

11. D'Amico TA. Undoing the gaps in quality, cost, and value. J Thorac Cardiovasc Surg. 2018;155:1211.

12. Tedesco G, Faggiano FC, Leo E, Derrico P, Ritrovato M. A comparative cost analysis of robotic-assisted surgery versus laparoscopic surgery and open surgery: the necessity of investing knowledgeably. Surg Endosc. 2016;30: 5044-51.

13. Paul S, Jalbert J, Isaacs AJ, Altorki NK, Isom OW, Sedrakyan A. Comparative effectiveness of robotic-assisted vs thoracoscopic lobectomy. Chest. 2014;146: 1505-12.

14. Park BJ, Flores RM. Cost comparison of robotic, video-assisted thoracic surgery and thoracotomy approaches to pulmonary lobectomy. Thorac Surg Clin. 2008; 18:297-300. vii.

15. Nasir BS, Bryant AS, Minnich DJ, Wei B, Cerfolio RJ. Performing robotic lobectomy and segmentectomy: cost, profitability, and outcomes. Ann Thorac Surg. 2014;98:203-8; discussion 208-9.

16. Swanson SJ, Meyers BF, Gunnarsson CL, Moore M, Howington JA, Maddaus MA, et al. Video-assisted thoracoscopic lobectomy is less costly and morbid than open lobectomy: a retrospective multiinstitutional database analysis. Ann Thorac Surg. 2012;93:1027-32.

17. General Thoracic Surgery Database v.2.3 -Training Manual. Available at: https:// www.sts.org/sites/default/files/documents/GTSD_V2_3_TrainingManual_June2018. pdf. Accessed July 2, 2018.

18. Kozower BD, Sheng S, O’Brien SM, Liptay MJ, Lau CL, Jones DR, et al. STS database risk models: predictors of mortality and major morbidity for lung cancer resection. Ann Thorac Surg. 2010;90:875-81; discussion 881-3.

19. McKenna RJ Jr, Houck W, Fuller CB. Video-assisted thoracic surgery lobectomy: experience with 1,100 cases. Ann Thorac Surg. 2006;81:421-5; discussion 425-6.

20. Kneuertz PJ, Singer E, D’Souza DM, Moffatt-Bruce SD, Merritt RE. Postoperative complications decrease the cost-effectiveness of robotic-assisted lobectomy. Surgery. 2019;165:455-60.

21. Singer E, Kneuertz PJ, D'Souza DM, Moffatt-Bruce SD, Merritt RE. Understanding the financial cost of robotic lobectomy: calculating the value of innovation? Ann Cardiothorac Surg. 2019 [Epub ahead of print].

22. Austin PC, Stuart EA. Moving towards best practice when using inverse probability of treatment weighting (IPTW) using the propensity score to estimate causal treatment effects in observational studies. Stat Med. 2015;34: 3661-79.

23. Geller AD, Zheng H, Mathisen DJ, Wright CD, Lanuti M. Relative incremental costs of complications of lobectomy for stage I non-small cell lung cancer. $J$ Thorac Cardiovasc Surg. 2018;155:1804-11.

24. Burfeind WR Jr, Jaik NP, Villamizar N, Toloza EM, Harpole DH Jr, D'Amico TA. A cost-minimisation analysis of lobectomy: thoracoscopic versus posterolateral thoracotomy. Eur J Cardiothorac Surg. 2010;37:827-32.

25. Watson TJ, Qiu J. The impact of thoracoscopic surgery on payment and health care utilization after lung resection. Ann Thorac Surg. 2016;101:1271-9; discussion 1979-80.

Key Words: robotic, thoracoscopic, VATS, minimally invasive, lobectomy, cost, value 


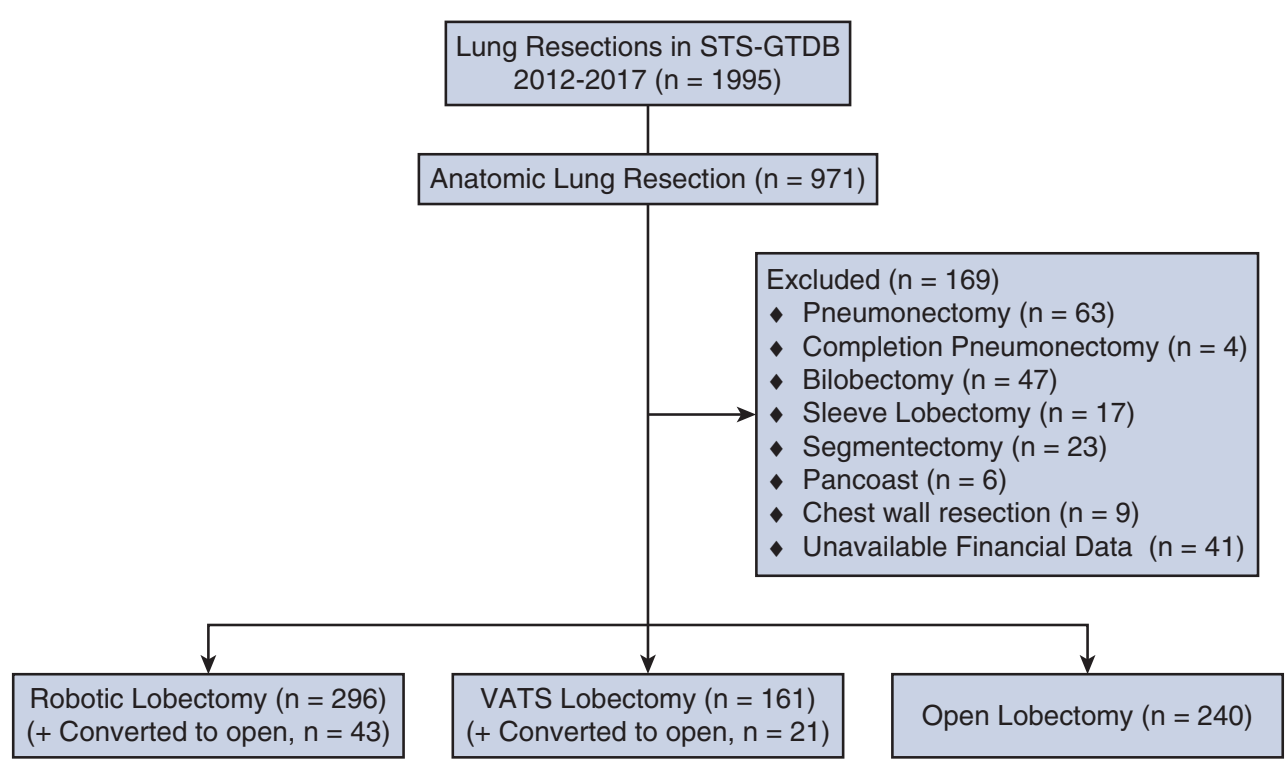

FIGURE E1. Consolidated Standards of Reporting Trials flowsheet outlining the selection of the study cohort. STS-GTSD, Society of Thoracic Surgery General Thoracic Surgery Database; Robotic, robotic-assisted thoracoscopic surgery; VATS, video-assisted thoracoscopic surgery; Open, open thoracotomy approach.

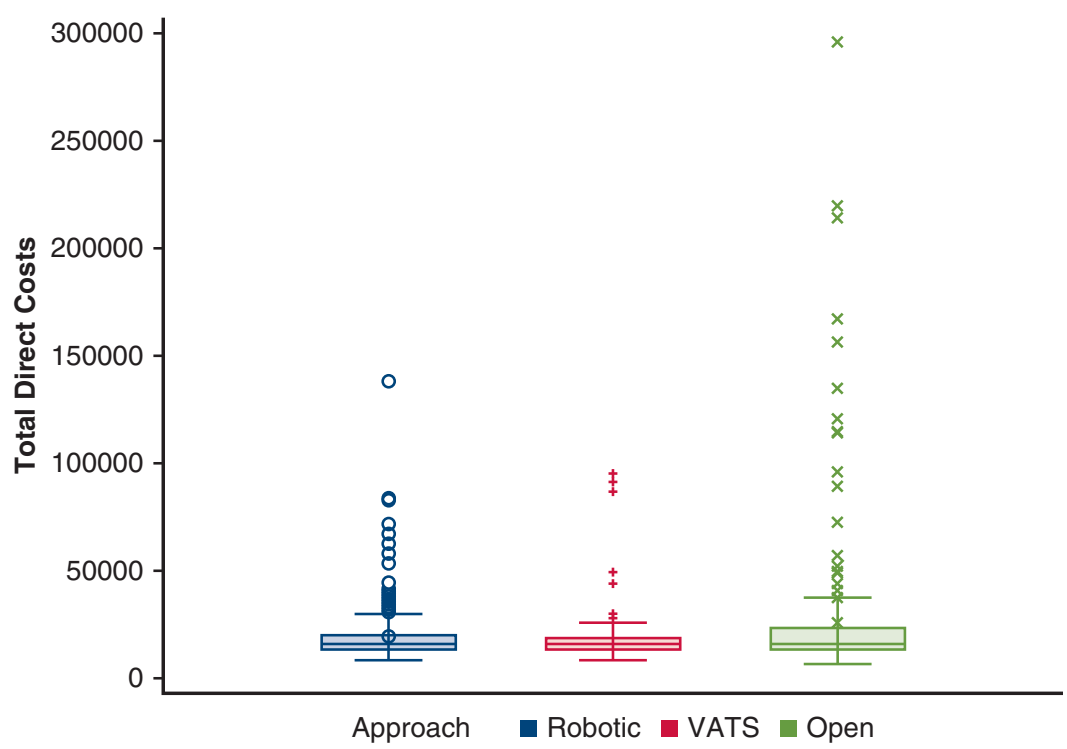

FIGURE E2. Distribution and variability of total direct cost for lobectomy by robotic, VATS, and open thoracotomy approach. In the box plots, costs are expressed in US dollars. The boundary of the box closest to zero indicates the 25th percentile, a line within the box marks the median, and the boundary of the box farthest from zero indicates the 75th percentile. Whiskers above and below the box indicate the 10th and 90th percentiles. Points (circle, cross, or $x$ ) above the whiskers indicate outliers. Robotic, Robotic-assisted thoracoscopic surgery; VATS, video-assisted thoracoscopic surgery; Open, open thoracotomy approach. 
TABLE E1. Unadjusted cost comparison by surgical approach

\begin{tabular}{|c|c|c|c|c|c|c|}
\hline & \multicolumn{3}{|c|}{ Mean cost $(95 \%$ CI $)$, US \$ } & \multicolumn{3}{|c|}{$P$ value } \\
\hline & Robotic (N = 296) & VATS $(N=161)$ & Open $(N=240)$ & $\begin{array}{l}\text { Robotic vs } \\
\text { VATS }\end{array}$ & $\begin{array}{l}\text { Open vs } \\
\text { robotic }\end{array}$ & $\begin{array}{l}\text { Open vs } \\
\text { VATS }\end{array}$ \\
\hline Total direct costs* & $\begin{array}{c}17,154 \\
(16,482-18,034)\end{array}$ & $\begin{array}{c}16,150 \\
(15,264-17,088)\end{array}$ & $\begin{array}{c}19,404 \\
(17,974-20,948)\end{array}$ & .16 & .004 & $<.001$ \\
\hline $\begin{array}{l}\text { Total indirect } \\
\text { costs* }\end{array}$ & $\begin{array}{c}17,320 \\
(16,613-18,058)\end{array}$ & $\begin{array}{c}15,409 \\
(14,505-16,370)\end{array}$ & $\begin{array}{c}18,024 \\
(16,825-19,308)\end{array}$ & .007 & .30 & $<.001$ \\
\hline $\begin{array}{l}\text { Operating room } \\
\text { costs } \dagger\end{array}$ & $\begin{array}{c}9882 \\
(9541-10,223)\end{array}$ & $\begin{array}{c}9528 \\
(8948-10,108)\end{array}$ & $\begin{array}{c}9064 \\
(8399-9730)\end{array}$ & .45 & .02 & .33 \\
\hline Total charges* & $\begin{array}{c}118,840 \\
(114,168-123,701)\end{array}$ & $\begin{array}{c}117,564 \\
(112,150-123,240)\end{array}$ & $\begin{array}{c}129,162 \\
(120,291-138,685)\end{array}$ & .80 & .02 & .03 \\
\hline
\end{tabular}

Values in bold indicate statistically significant $P$ values. CI, Confidence interval; Robotic, robotic-assisted thoracoscopic surgery; VATS, video-assisted thoracoscopic surgery; Open, open thoracotomy approach. Multiple comparisons of cohort differences were adjusted for using the Tukey-Kramer method. "Geometric mean and $95 \%$ confidence interval $(95 \% \mathrm{CI})$ based on log scale values transformed back to costs expressed as US dollar. †Operating room costs data were only available from 2012 to $2015 ; 518$ cased are included (robotic $=227$, VATS $=84$, and open $=207$ )

TABLE E2. Propensity score-adjusted cost comparison by surgical approach, intention-to-treat analysis*

\begin{tabular}{|c|c|c|c|c|c|c|}
\hline \multirow[b]{2}{*}{ Cost } & \multicolumn{3}{|c|}{ Mean cost $(95 \%$ CI $)$, US \$ } & \multicolumn{3}{|c|}{$P$ value } \\
\hline & Robotic $(\mathbf{N}=339)$ & VATS $(\mathbf{N}=182)$ & Open $(N=240)$ & $\begin{array}{l}\text { Robotic vs } \\
\text { VATS }\end{array}$ & $\begin{array}{l}\text { Open vs } \\
\text { robotic }\end{array}$ & $\begin{array}{l}\text { Open vs } \\
\text { VATS }\end{array}$ \\
\hline Total direct costs $\dagger$ & $\begin{array}{c}17,743.1 \\
(16,963.1-18,559.0)\end{array}$ & $\begin{array}{c}18,102.4 \\
(16,106.2-20,345.9)\end{array}$ & $\begin{array}{c}18,079.2 \\
(16,877.0-19,367.0)\end{array}$ & .95 & .90 & $>.99$ \\
\hline $\begin{array}{l}\text { Total indirect } \\
\text { costs } \dagger\end{array}$ & $\begin{array}{c}17,694.0 \\
(16,934.2-18,487.8)\end{array}$ & $\begin{array}{c}16,730.2 \\
(14,927.6-18,750.6)\end{array}$ & $\begin{array}{c}17,064.5 \\
(15,994.6-18,206.0)\end{array}$ & .64 & .63 & .95 \\
\hline $\begin{array}{l}\text { Operating room } \\
\text { costs } \ddagger\end{array}$ & $\begin{array}{c}10,049.5 \\
(9682.6-10,416.3)\end{array}$ & $\begin{array}{c}9472.6 \\
(8701.1-10,244.2)\end{array}$ & $\begin{array}{c}8674.8 \\
(8158.8-9190.8)\end{array}$ & .38 & $<.01$ & .21 \\
\hline Total charges $\dagger$ & $\begin{array}{c}122,960.7 \\
(118,171.7-127,943.7)\end{array}$ & $\begin{array}{c}12,8967.1 \\
(117,122.8-142,009.1)\end{array}$ & $\begin{array}{c}121,171.5 \\
(113,656.3-129,183.5)\end{array}$ & .64 & .92 & .54 \\
\hline
\end{tabular}

CI, Confidence interval; Robotic, robotic-assisted thoracoscopic surgery; VATS, video-assisted thoracoscopic surgery; Open, open thoracotomy approach. *Multiple Comparisons of cohort differences were adjusted for using the Tukey-Kramer method. †Geometric mean and $95 \%$ confidence interval ( $95 \% \mathrm{CI})$ based on log scale values transformed back to costs expressed as US dollar. ‡Operating room costs data were only available from 2012 to 2015; 518 cased are included (robotic $=263$, VATS $=103$, and open $=207$ ). 